\title{
BioéthiqueOnline
}

\section{Le corps humain converti en gisement de ressources économiques - À propos de Le Corps-Marché, de Céline Lafontaine}

\section{Danielle Moyse}

Volume 4, 2015

Reçu : 28 Feb 2015; publié : 17 Mar 2015; éditrices : Lise Lévesque \& Aliya Affdal

URI : https://id.erudit.org/iderudit/1035493ar

DOI : https://doi.org/10.7202/1035493ar

Aller au sommaire du numéro

Éditeur(s)

BioéthiqueOnline

ISSN

1923-2799 (numérique)

Découvrir la revue

Citer cet article

Moyse, D. (2015). Le corps humain converti en gisement de ressources économiques - À propos de Le Corps-Marché, de Céline Lafontaine. BioéthiqueOnline, 4. https://doi.org/10.7202/1035493ar
Résumé de l'article

Le livre de Céline Lafontaine propose, à partir du recensement de travaux de chercheurs francophones et anglo-saxons, une description de la marchandisation de la vie humaine à l'ère de la bioéconomie, ainsi que le mentionne le sous-titre du livre. Le texte ci-dessous en présente un résumé ainsi qu'une mise en perspective philosophique du phénomène que montre l'auteure. 


\title{
Le corps humain converti en gisement de ressources économiques - À propos de Le Corps-Marché, de Céline Lafontaine
}

\author{
COMMENTAIRE / COMMENTARY \\ Danielle Moyse ${ }^{1}$
}

Reçu/Received: 28 Feb 2015

Éditrices/Editors: Lise Lévesque \& Aliya Affdal

Publié/Published: 17 Mar 2015

2015 D Moyse, Creative Commons Attribution 4.0 International License

\begin{abstract}
Résumé
Le livre de Céline Lafontaine propose, à partir du recensement de travaux de chercheurs francophones et anglo-saxons, une description de la marchandisation de la vie humaine à l'ère de la bioéconomie, ainsi que le mentionne le sous-titre du livre. Le texte ci-dessous en présente un résumé ainsi qu'une mise en perspective philosophique du phénomène que montre l'auteure.
\end{abstract}

\section{Mots clés}

corps humain, éthique, bioéconomie, marchandisation, objectivation, technosciences, dignité, Céline Lafontaine

\section{Summary}

Building on a review of the work of French and AngloSaxon researchers, Céline Lafontaine's book provides a description of the commodification of human life in the era of the bioeconomy, as mentioned in the subtitle of the book. The text below is a summary and a philosophical perspective of the phenomenon presented by the author shows.

\section{Keywords}

human body, ethics, bioeconomy, commodification, objectification, technosciences, dignity, Céline Lafontaine

Affiliations des auteurs / Author Affiliations

${ }^{1}$ Professeur agrégé de philosophie à L'éducation nationale, chercheuse associée CNRS-INSERM, Docteur en philosophie, Paris, France

\section{Correspondance / Correspondence}

Danielle Moyse, danielle.moyse@orange.fr

\section{Remerciements}

Je remercie BioéthiqueOnline pour la place accordée à l'évocation des difficultés éthiques que soulève l'ouvrage très instructif de Céline Lafontaine, et en particulier Lise Lévesque pour l'échange fructueux qui a donné lieu à cette collaboration.

\section{Conflit d'intérêts}

Aucun déclaré

\section{Acknowledgements}

Thanks to BioéthiqueOnline for the place given to highlighting the ethical difficulties raised by Céline Lafontaine's very informative book, and especially to Lise Lévesque for the fruitful exchange that led to this collaboration.

\section{Conflicts of Interest}

None to declare

Ce commentaire complète une vidéo intitulée Ethique \& finitude: le corps humain comme gisement de ressources, disponible sur le site Philosophies.tv.

\section{Introduction}

Dans le texte qu'il consacra en 1954 à " la question de la technique », Martin Heidegger évoque la provocation très particulière par laquelle « la nature est désormais mise en demeure de livrer une énergie qui puisse, comme telle, être extraite et accumulée. " [1, p.20] Dans cet horizon, tout est sommé de nous apparaître comme gisement de ressources potentielles. Là où le paysan d'autrefois " confiait la semence aux forces de croissances et veillait à ce qu'elle prospère » [1, p.21], la culture des champs est aujourd'hui prise

dans le mouvement aspirant d'un mode de culture d'un autre genre qui requiert la nature. II la requiert au sens de la provocation. L'agriculture est aujourd'hui une 
industrie d'alimentation motorisée. L'air est requis pour la fourniture d'azote, le sol pour celle de minerais, le minerai par exemple pour celle d'uranium, celui-ci pour celle d'énergie atomique, laquelle peut être libérée pour des fins de destruction ou pour une utilisation pacifique [1, p.21].

Au cours de ce mouvement général de sommation, l'ensemble des énergies naturelles est converti en un gigantesque stock dans lequel il devient possible de puiser indéfiniment. Mais le penseur se demande aussitôt si, dans un tel processus, l'homme n'est pas impliqué de telle manière qu'il fasse lui-même partie du fonds disponible : " la façon dont on parle couramment de matériel humain, de l'effectif des malades d'une clinique, le laisserait penser », précise-t-il [1, p.24].

Mais à en croire Le Corps-Marché, le livre par lequel Céline Lafontaine a recensé les travaux effectués par des chercheurs anglo-saxons et francophones sur la marchandisation du corps humain, le fait que celui-ci soit désormais intégralement converti en gisement de ressources au service du dispositif technico-économique qui semble désormais s'imposer à nous ne laisse plus place au moindre doute. "Les questions d'ordre éthique, prévient pourtant la sociologue, ont volontairement été laissées de côté afin de mettre l'accent sur les mécanismes économiques, politiques et sociologiques qui sous-tendent le corps-marché. » [2, p.14] Mais la présentation critique qui suit fait apparaître, à elle seule, l'enjeu éthique de l'ouvrage de Céline Lafontaine : comment le corps humain pourrait-il devenir une marchandise parmi d'autres sans que le séjour humain (éthique, renvoyant à ethos qui signifie « séjour »!) en soit sérieusement altéré?

\section{Le retournement du projet de la « bioéconomie »}

Céline Lafontaine montre comment une telle marchandisation s'est opérée dans l'horizon de ce que Nicholas Georgescu Roegen a nommé, en 1975, la « bioéconomie ». Mais là où cet économiste avait tenté d'élaborer, sous un pareil concept, un modèle de développement économique qui tienne compte des limites du vivant, s'est opéré, notamment sous la pression de l'Organisation de Coopération et de Développement Économique (OCDE) un renversement qui a abouti à l'instauration d'une économie où l'ensemble du vivant, en particulier le corps humain, est devenu une source de productivité [2, p.33]. Laquelle est supposée au service d'une croissance illimitée, elle-même légitimée par la promesse d'une régénération des corps.

Plus aucun élément du corps n'échappe alors au processus : le sang dont le stockage ouvrit la porte à celui de toutes les autres substances corporelles, les lignées cellulaires, le sperme, les ovocytes, les organes, les prépuces de bébés circoncis (!), les embryons, etc. Alors que les législations internationales proclament solennellement, et ce, en mémoire du code de Nuremberg, "l'indisponibilité du corps humain », le livre de Céline Lafontaine et les travaux sur lesquels il s'appuie montrent notamment comment se profile une exploitation du corps des plus pauvres au nom même de cette indisponibilité! Ainsi, la sociologue évoque-t-elle l'histoire d'Henrietta Lacks, jeune femme afro-américaine morte d'un cancer de l'utérus en 1951 et dont les descendants découvrirent des décennies plus tard que des chercheurs avaient fait dériver des cellules de leur mère une importante lignée cellulaire humaine qui est au cœur de la recherche médicale depuis les années cinquante. Or, " le contraste entre la pauvreté des enfants d'Henrietta Lacks et le prestige de la lignée cellulaire obtenue grâce à la maladie de leur mère apparaît d'autant plus frappant », écrit Céline Lafontaine, "lorsqu'on sait que ces cellules ont permis aux entreprises qui les ont commercialisées d'engranger des profits considérables, sans qu'un seul dollar ne soit versé à la famille. » [2, p.93] Le cas de John Moore, jeune homme atteint d'une forme rare de leucémie est tout aussi éloquent : ayant subi une ablation de la rate en 1976, il s'aperçut en 1981 que le médecin qui avait suivi son cas avait obtenu un brevet pour une lignée cellulaire dérivant de ses cellules cancéreuses d'une valeur de 15 millions de dollars, fruit d'un accord entre le chercheur, un institut de recherche génétique et une entreprise pharmaceutique. Choqué qu'on ait pu commercialiser ses cellules sans son consentement, John Moore entama en vain une procédure judiciaire au terme de 
laquelle ne lui fut accordée aucune compensation financière [2, p.95-96]. Le motif invoqué pour justifier un tel verdict fut qu'il ne fallait pas de risquer d'entraver les avancées de la recherche en donnant aux pourvoyeurs des "matériaux de recherche », un droit de regard sur ces derniers! D'ailleurs, ces événements ont forcé la communauté scientifique à mieux encadrer la recherche et c'est maintenant la règle d'informer les participants à la recherche des possibilités de commercialisation des dérivés biologiques. Ce processus de consentement et information, bien que souhaitable, ne garantit toutefois pas que les plus démunis ne soient pas poussés par le besoin (d'argent ou autre avantage) à participer à des recherches.

\section{La perversion des principes destinés à protéger la dignité humaine}

Les raisons du malaise provoqué par la lecture du livre de Céline Lafontaine, tiennent à la fois au caractère profondément injuste de la marchandisation du corps qui y est décrite, à sa violence et à sa dimension perverse. Même si nous ne mentionnions pas les dérives insoutenables qui conduisent parfois des hommes et des femmes frappés par la misère à vendre un de leurs organes (Céline Lafontaine rappelant les travaux des chercheurs qui ont mis en évidence que, lors du tsunami de 2004, des femmes se sont vues contraintes de sacrifier un de leurs reins pour nourrir leur famille!), il suffit de rappeler la dimension honteusement inégalitaire d'un système de santé où, par exemple, $70 \%$ des transplantations rénales ont, en 2007, été effectuées en Amérique du Nord et dans l'Union européenne [2, p.82]. Apparaît sans contestation possible le fait que la marchandisation du corps se fait " au bénéfice " des plus riches, pour autant que qui que ce soit puisse se sentir épargné par un dispositif dont l'énumération des principaux aspects provoque, purement et simplement, la nausée. Même au sein des pays les plus favorisés économiquement, ce sont les plus fragiles de ces pays-là qui font l'objet d'une sournoise exploitation, et concernant la médecine de la procréation, Céline Lafontaine n'hésite pas à écrire qu'elle a «transformé le corps féminin en usine à produire des ovules » [2, p.163] Le règne de l'efficience totale n'atteint-il pas, dans cette perspective, l'humanité dans sa dignité même?

D'autant plus que, paradoxalement, il semble se déployer sous l'égide de la rhétorique du don! C'est notamment le cas pour ce qui concerne le "marché de la procréation » qui a donné lieu au développement de ces "banques de sperme qui ont marqué le premier pas vers la mise en ressource et la commercialisation des cellules reproductives » [2, p.148], tandis que, dans le même temps, " la répugnance morale à associer la procréation humaine à un marché a favorisé le transfert du modèle du don à l'industrie de la procréation. " [2, p.148] Ainsi, " même dans les pays où la vente et l'achat de sperme sont légalisés comme les États-Unis, l'expression " donneurs » est utilisée pour désigner les hommes qui donnent volontairement aux banques de sperme » [2, p.148]. De même, comme le «don » d'ovocytes suscite paradoxalement une "compensation financière », il attire particulièrement aux États-Unis de jeunes étudiantes qui voient dans cette compensation le moyen de financer leurs études [2, p.149].

Dans la même logique, l'exploitation des plus pauvres se fait-elle au nom de la protection de la dignité humaine car « si un individu ne peut, au nom de la dignité, vendre son corps, entier ou en parties, il est toutefois permis à d'autres (chercheurs, laboratoires pharmaceutiques) d'acquérir des droits d'exploitation commerciale pour des éléments corporels sur la base d'une valorisation juridique de leur travail. » [2, p.109]

Plus gravement encore, c'est à une perversion du principe suivant lequel une intervention sur un corps humain n'est légitime que si elle a lieu avec "le consentement éclairé » de l'intéressé, que semble bel et bien donner lieu la marchandisation évoquée par le livre de Céline Lafontaine. Par exemple, « l'imposition de la norme libérale d'un individu libre et consentant contraste fortement avec les réalités sociales et culturelles des patients indiens qui n'ont bien souvent que leur corps biologique comme ressource économique et qui ont rarement accès à des soins de santé de qualité. » [2, p.219] Au bout du compte, le « consentement éclairé » en vient à servir de caution à l'emprise économique 
sur la vie humaine plutôt qu'à protéger les plus démunis : ces derniers auront consenti aux diverses interventions sur leur propre corps, mais auront-ils eu le choix de ne pas consentir?

\section{La généalogie du phénomène de la marchandisation du corps}

Reste évidemment à comprendre comment nous avons pu aboutir à une pareille situation, et, par conséquent, à tenter la généalogie du phénomène. Nous pouvons à la fois en proposer la genèse technique et historique, mais, plus radicalement, examiner les fondements philosophiques qui ont ouvert la porte à une telle dérive.

Céline Lafontaine rappelle que l'exploitation du corps humain a eu de lourds précédents dans l'histoire des hommes, et s'est fâcheusement illustrée par l'esclavage. Mais pour que les éléments du corps humain aient progressivement pu constituer un gisement de ressources économiques illimitées, il a fallu des avancées techniques et scientifiques qui n'étaient pas nécessaires à la commercialisation des esclaves. "L'utilisation biomédicale de la matière corporelle repose sur un long processus d'objectivation dont les fondements sont à rechercher du côté des origines de la médecine moderne » [2, p.71] écrit Céline Lafontaine. S'appuyant notamment sur les travaux de David le Breton ou de David Chamayou, la sociologue rappelle alors le rôle de la dissection des cadavres dans la marchandisation des corps : "L'ouverture des corps et la transgression des tabous entourant leur traitement ont constitué une étape importante dans le processus d'objectivation amorcé par la médecine moderne. " [2, p.71] Le cadavre sera donc le premier à constituer la " matière première » de la recherche médicale et du dispositif économique et technique dans lequel cette marchandisation va se déployer.

Un autre élément décisif consiste en la mise au jour des processus internes des organismes : en 1907, Harrison produit une première lignée cellulaire de grenouille qui rend visible au regard humain ce qui lui était auparavant caché, tout en mettant en retrait l'organisme lui-même. Ce qui était invisible devient visible, tandis que le corps lui-même tend à être éclipsé par la découverte de son fonctionnement biologique.

S'opère alors une déréalisation du corps sans laquelle l'ensemble de sa marchandisation ne serait guère possible. Car ce n'est plus tant le corps lui-même qui est marchandisé que l'ensemble de ses constituants : sang, cellules, gamètes, ovocytes, etc. Les embryons humains sont pris dans le processus, mais ils ne le sont pas en tant qu'embryons, mais en tant que " matière première » de la procréation, ou plus exactement de la reproduction. Les aspects techniques, médicaux et historiques du phénomène ne suffisent toutefois pas à en rendre compte.

En effet, la déréalisation médicale et technique du corps n'est peut-être que la concrétisation d'une déréalisation qui tient aux fondements philosophiques même de notre histoire. Et je me permettrai ici de mettre le travail de Céline Lafontaine avec d'autres éléments susceptibles d'éclairer le phénomène qu'elle décrit. Ainsi, le livre d'Ingrid Auriol paru récemment sous le titre Intelligence du corps [3] montre-t-il à quel point nous ne sommes précisément pas en intelligence, non plus qu'en amitié avec lui. Plus radicalement, nous avons même oublié de quelle intelligence le corps est porteur. L'olfaction, le goût sont superbement ignorés par la plupart des philosophes. Par exemple, Platon peut-il noter dans Hippias majeur que ne peut être jugé beau ce qui relève du goût ou de l'odeur. La philosophie de Descartes comporte certes des réflexions sur l'union de l'âme et du corps, mais, après avoir obtenu la certitude de son existence, le philosophe répond à la question « qui suis-je? », par : " une chose qui pense. " Et cette " chose » qui pense, c'est l' " âme » et non le corps!

Que le corps sache ce que nous ne savons plus ou ne savons pas encore, il nous faudra attendre la phénoménologie et la littérature contemporaine pour nous en apercevoir. Ainsi, Ingrid Auriol rappellet-elle que [3, p.74], dans l'Introduction à la métaphysique, Heidegger note qu'un ancien lycée n'est pas le même pour ceux qui le voient de l'extérieur et pour les élèves qui sont assis à l'intérieur, et 
qu'on garde souvent l'odeur des vieux bâtiments dans les narines au bout de plusieurs décennies, avant de conclure que cette odeur "nous restitue l'être de ce bâtiment d'une façon beaucoup plus immédiate et véritable qu'aucune description ou visite ne saurait le faire. » [4, p.45].

Plus globalement encore, la philosophie a bel et bien tendance à se tenir à distance de tout ce qui engage profondément notre corps, ainsi qu'en témoigne également l'affirmation kantienne d'après laquelle ce qui est beau dans une peinture, c'est le dessin et non la couleur, qui relève encore des attraits et de la sensation. La définition par Kant de la beauté comme " plaisir désintéressé " le conduit à renier du rapport au monde, tout ce qui nous y engage corporellement de façon profonde [5, p.67].

Quoi qu'il en soit, l'abrogation technique et scientifique du corps décrite par le livre de Céline Lafontaine trouve ses conditions de possibilité philosophiques tout au long d'une histoire de l'Occident au cours de laquelle l'homme se tient à distance du corps, bien avant que la science l'ait annulé dans sa corporéité la plus vive. Et si, pour rendre compte du phénomène, décrit minutieusement par Céline Lafontaine, de la conversion du corps en objet, il nous faut sonder les présupposés philosophiques de l'Occident, c'est bien sûr vers la philosophie de Descartes qu'il convient à nouveau de se tourner. Car cette pensée met un terme à la philosophie médiévale qui conçoit l'homme comme " enfant de Dieu » pour faire de lui le sujet pensant. Terme qu'il faut entendre avec toute la rigueur philologique qui convient, à savoir comme le sub-jectum, c'est-à-dire comme ce qui est jeté dessous une chose pour lui servir de fondement. Tel est l'homme des temps nouveaux : jeté dessous toute chose pour lui servir de base. Ce qui éclaire ici notre problème à tous égards! Car le sujet, l'homme/fondement, ne peut qu'être en quête de cette santé illimitée dont parle Céline Lafontaine et qui est supposée légitimer la conversion du corps en objet de recherche, avant qu'il ne se transforme en pure et simple marchandise : comment ce sujet, qui est au fondement de tout, pourrait-il être faillible?

Et, en l'occurrence, ce n'est plus même la santé, mais la régénération des corps qui est visée. II ne faut pas oublier que le précédent livre de Céline Lafontaine porte sur ce qu'elle a appelé La société post-mortelle [6]. Car c'est bel et bien en un tel déni de finitude que s'origine la marchandisation des corps dont parle la sociologue. Sur la base d'un tel déni, peut alors se déployer l'objectivation des corps qui n'est que la suite logique de l'avènement de l'homme/sujet. Puisque ce qui fait face au sujet, c'est, en toute cohérence : l'objet. Installé dans le monde à titre de sujet, l'homme ne peut que chercher à convertir tout ce auprès de quoi il se trouve en objet. Le monde du sujet ne peut plus être qu'un monde d'objets. Ce qui n'implique guère d'amitié vis-à-vis des objets en question. Car l'objet n'est, en tant que tel, qu'un élément adverse et qui me fait face, ainsi que le mot allemand Gegen-stand le fait plus lumineusement apparaître. L'objet est debout contre ou, en français, face au sujet qui tentera d'exercer sur lui son pouvoir. C'est pourquoi la conversion de toute chose en objet est le prélude à la considération de l'ensemble de ce qui est en fonds indéfiniment disponible, c'est-àdire en stock à notre disposition.

Dans cet horizon, les philosophies utilitaristes de Bentham et de Mill mentionnées par Céline Lafontaine peuvent servir d'activateur ultime à la marchandisation du corps.

Avec l'extension de la science médicale au XIXème siècle, la quête de nouveaux corps disponibles pour la dissection devient un enjeu social. C'est dans ce contexte que le philosophe Jeremy Bentham décide d'offrir son corps à la science, dans l'espoir d'encourager les gens de toutes les classes sociales à suivre son exemple. Étroitement lié au libéralisme économique, l'utilitarisme philosophique a ainsi contribué à légitimer la mise en ressource du corps humain (...) Cette rencontre de la science médicale et de l'utilitarisme économique au XIXème siècle a marqué une étape importante du processus d'objectivation du corps. [2, p.73] 


\section{Des questions humainement décisives soulevées par la marchandisation du corps}

Le livre de Céline Lafontaine qui fait un état des lieux sans concession de l'emprise du dispositif économique, technique et scientifique sur ce qu'on a un peu de difficulté à nommer encore "le corps humain " pose en réalité des questions d'une redoutable difficulté concernant les limites des interventions légitimes sur le corps. Ces interventions semblent se déployer avec un tel cynisme qu'on peut être amené à se demander si un rapport juste et sain aux fulgurants progrès techniques qui sous-tendent la "bioéconomie " est possible. Les législations nationales ou internationales (qui ne sont guère évoquées de façon détaillée dans le livre) ont-elles le moindre pouvoir de régulation sur ce qui est en cours? Individuellement, sommes-nous fatalement impliqués à notre insu dans un phénomène entièrement pervers? Par exemple, si l'état de santé de l'un de nous ou de nos proches nécessite une greffe, entretenons-nous un tel système? S'il y a pourtant des femmes et des hommes qui donnent en toute générosité leur sang, leur moelle osseuse, leurs organes, à quelles conditions incontournables cela peut-il être, malgré tout, légitime? À quelles interventions scientifiques sur le corps faut-il absolument résister pour préserver la dignité humaine?

Plus radicalement encore, ne sommes-nous pas invités, si l'on en juge par le terrible descriptif de la marchandisation du corps proposé par Céline Lafontaine, à repenser fondamentalement notre rapport à la santé, présentée par la techno-science comme un idéal exempt de tous maux, et qui finirait si possible par éradiquer la mort ? Car l'ouvrage de Céline Lafontaine semble bien administrer la preuve de ce que Joé Bousquet, le poète demeuré paralysé après qu'il eut reçu une balle dans la colonne vertébrale pendant la guerre de 1914, déclare dans ses superbes Lettres à Poisson d'Or: "Ce qu'il y a de diminuant pour une créature mortelle, ce n'est pas qu'elle doive mourir, mais qu'elle vive comme expulsée de cette mort qui la caractérise, (...), qu'elle ne se rende la vie supportable qu'en en éliminant mentalement le caractère essentiel qui est justement la mort à venir. » [7, p.48] Est-ce à dire qu'à l'heure où l'on n'en est plus simplement à l'élimination " mentale » du caractère essentiellement mortel de la vie humaine, dont parle le poète, mais à une tentative effective de victoire sur la mort, cette aspiration est la plus « diminuante » que nous pourrions concevoir?

Dans cet horizon que penser, par exemple, de la conception d'enfants après la mort de leurs parents " grâce " au stockage des "matériaux " nécessaires à la procréation, qui témoigne de la volonté claire de dépasser les limites temporelles assignées à l'existence humaine? « Occupant la place jadis réservée à l'âme dans le christianisme », déclare ainsi Céline Lafontaine, "les gènes sont alors perçus comme la source de l'immortalité terrestre, comme ce qui se réincarne une fois la vie corporelle achevée. »[6, p.106] N'est-ce pas expulser purement et simplement l'homme de son séjour terrestre?

\section{Références}

1. Heidegger, M., La question de la technique. Dans: Essais et conférences. Paris; Gallimard; Collection Tel (no. 52); 1980. (Première parution en 1958. Traduit de l'allemand par André Préau.)

2. Lafontaine, C., Le Corps-Marché, La marchandisation de la vie humaine à l'ère de la bioéconomie, Paris; Éditions du Seuil; 2014.

3. Auriol, I., Intelligence du corps, Éditions du Cerf; Collection « La Nuit surveillée »; 2013.

4. Heidegger, M., Introduction à la métaphysique, Paris; Gallimard; Collection Tel ( $\left.n^{\circ} 49\right) ; 1967$. (Première parution en 1967. Traduit de l'allemand par Gilbert Kahn.)

5. Kant, E., Critique de la faculté de juger, I, §14, Paris; Éditions Vrin; 1984.

6. Lafontaine, C., La société post-mortelle, Paris; Éditions du Seuil; 2008.

7. Bousquet, J., Lettres à Poisson d'Or, Paris; Gallimard; Collection L'Imaginaire ( $\left.n^{\circ} 198\right) ; 1967$. 